\title{
Staphylococcus aureus recovery from cotton towels
}

\author{
Anna R. Oller and Ashley Mitchell \\ Department of Biology and Earth Science, University of Central Missouri
}

\begin{abstract}
Background: Staphylococcus aureus is an emerging pathogen afflicting healthy individuals without known risk factors, and methicillinresistant Staphylococcus aureus has been shown to colonize multiple family members sharing households. Because household items such as towels are often shared by family members, this study investigated whether cotton towel absorbency or washing conditions affect Staphylococcus aureus cell viability or cell retention, and whether the levels may be sufficient for person-to-person transmission.

Methodology: Staphylococcus aureus ATCC 25923 was added to a $48 \mathrm{~mm}^{2}$ template area on three cotton towel types (terry, pima, and Egyptian), and subjected to hand washing, without manual wringing, in three conditions (water only, bleach addition, or liquid detergent addition). Serial dilutions plated onto mannitol salt plates quantified bacteria for inoculations, pre- and post-wash water samples, towel surfaces, and hand transfer. Hand transfer of bacteria was determined on towels immediately, one, 24, and 48 hours post inoculation.

Results: Bleach $(p \leq .05)$ was the most effective at reducing bacterial viability on all towel types compared to detergent and water. Although not statistically significant, more Staphylococcus colonies were recovered from higher absorbency towels and from inside directly inoculated template areas. A paired $t$-test showed a difference between immediate and one-hour CFUs versus 24- and 48-hour recoveries (0.0002) for hand transfers.

Conclusions: Cell viability decreased for over 48 hours on towels, but sufficient quantities may remain for colonization. More absorbent towels may harbor more Staphylococci than less absorbent ones, and may serve as a transmission mechanism for the bacterium.
\end{abstract}

Keywords: bacteria, bleach, infection, Staphylococcus, towels, transmission

J Infect Developing Countries 2009; 3(3):224-228.

Received 4 April 2008 - Accepted 7 January 2009

Copyright $\odot 2009$ Oller and Mitchell. This is an open-access article distributed under the Creative Commons Attribution License, which permits unrestricted use, distribution, and reproduction in any medium, provided the original work is properly cited.

\section{Introduction}

Staphylococcus aureus is emerging as a pathogen frequently colonizing and causing infections in healthy individuals without known risk factors such as a recent hospitalization or antibiotic usage. Risk factors for acquiring Staphylococcus infections include bars of soap and soap dispensers [1], towels [2], and direct skin contact [3]. Studies of methicillin-resistant Staphylococcus aureus (MRSA) showed transmission between members of 7 out of 10 families, as well as between different families [4-5]. Towels are reused several times before being washed and they are often shared among individuals.

A wound surface infected with Staphylococcus aureus can contain up to $3 \times 10^{8}$ Colony Forming Units (CFUs) $/ \mathrm{cm}^{2}$. Further, contaminated wound dressings can contain more than $10^{5}$ organisms [6]. Gardner et al. [7] determined that chronic wound infections contain approximately $10^{5}$ of $S$. aureus per gram of tissue, but another study [6] found a median of $3.4 \times 10^{3}$ bacteria in infected burn wounds. Actual bacterial levels required for skin colonization have not yet been determined.
Previous studies have demonstrated $S$. aureus transfer to surgical gowns [8], to polyester or cottonpolyester blend fabrics [9-11], and to fabrics subjected to detergent systems utilizing different washing temperatures [12]. Other studies [2,6,10,11] have shown $S$. aureus survival for days on various types of fabrics. No study has previously investigated the different absorbencies of towels on $S$. aureus recovery and how likely the bacterium will be transferred to hands. Our goal was to evaluate $S$. aureus cell retention and viability on three types of cotton towels subjected to three washing conditions. In addition, cell viability and hand transfer of bacteria on towels left at room temperature for up to 48 hours were investigated. This study was exempt from review by our internal review board.

\section{Materials and Methods}

A $1 \times 3 \times 3 \times 3$ experimental design was utilized for $S$. aureus cell retention and viability. The independent variables were the $S$. aureus, the $S$. aureus concentrations $\left(1.42 \times 10^{8}, 1.78 \times 10^{8}\right.$, and $2.75 \times 10^{8}$ ), towel type (terry: least absorbent; pima 
and Egyptian: most absorbent) and washing condition (water only, detergent addition, or bleach addition). The dependent variable was the $S$. aureus retention and viability. Autoclaved water served as the control.

The all-cotton towels (same company brand) were uniformly sized $(390 \mathrm{~cm} \times 320 \mathrm{~cm})$. They were weighed (terry $=64.88 \mathrm{~g}$; pima $=56.34 \mathrm{~g}$; and Egyptian $=76.65 \mathrm{~g}$ ), and tested for water absorbency $($ terry $=238 \mathrm{ml} ;$ pima $=245 \mathrm{ml} ;$ Egyptian $=273.5 \mathrm{ml})$ via displacement to determine absorbent classification.

\section{Culturing Bacteria}

Methicillin sensitive Staphylococcus aureus 29523 (Hardy Diagnostics, Phoenix, AZ) was chosen for this study because it was a clinical isolate and is commonly utilized in Staphylococcal research.

Staphylococcus aureus was inoculated into Tryptic Soy Broth tubes and incubated for 24 hours at $37^{\circ} \mathrm{C}$ similar to the procedure described by Wilkoff [2] to simulate a potentially contaminated surface with the bacterium. Staphylococcus aureus was added to a $48 \mathrm{~mm}^{2}$ center template area (identical area on each towel) of autoclaved (Tuttanauer, St. Louis, MO) all-cotton towels and allowed to absorb for one hour.

\section{Water and Washing Conditions}

The washing specifications of automated toploading washing machines were obtained to determine tub and water capacity, tub and agitation speed, spinning speed, and time frame of each cycle. One towel was added to each tub, and washed per the washing machine manufacturer's specifications. Towels were aseptically removed without wringing to prevent false bacterial addition or displacement, and replica plating was immediately performed.

A $1 \mathrm{ml}$ pre-wash sample (both with and without additives) was serially diluted onto mannitol salt plates to ensure no water, air, or additive contamination. Bleach $(20 \mathrm{ml})$ and liquid detergent $(100 \mathrm{ml})$ were chosen as additives since they are commonly employed when washing clothes. To determine if bleach and detergent supported the growth of bacteria, known concentrations were added to culture tubes containing each additive, serial dilutions were performed, and mannitol salt plates were inoculated and incubated at $37^{\circ} \mathrm{C}$. Liquid detergent containing a bleach additive was intentionally not utilized so we could test bleach independently. The water temperature ranged from $30^{\circ} \mathrm{C}$ initially, and subsequently cooled to $27^{\circ} \mathrm{C}$ by the end of the experiment. Lukewarm water was chosen intentionally since many people do not wash clothes in hot water.

The initial $\mathrm{pH}$ of the water was 6.7 , bleach was 11.69, and detergent was 13.0. Diluted bleach $\mathrm{pH}$ yielded 10.9 and the diluted detergent $\mathrm{pH}$ was 10.96 . The $\mathrm{pH}$ between the bleach and detergent used for testing was consistent, eliminating $\mathrm{pH}$ as a recovery factor.

\section{Towel Inoculation and Washing}

Towels were divided into two groups, one for viability and hand transfer determination, and one for washing conditions. The towels used for the viability test were covered with sterile aluminum foil to prevent aerosol contamination, and left at $25^{\circ} \mathrm{C}$ to simulate towels being left after bacterial contact. A $10 \times 5.5 \mathrm{~cm}$ replica plater (Fisher Scientific, Pittsburgh, PA) was pressed against the towels for three seconds to obtain direct contact samples of the towels, both within and outside of the template area to determine bacterial retention and transfer during washing. Sterile water $(0.1 \mathrm{ml})$ was then added to the template area using a serological pipette and pipetted up and down three times and plated to evaluate how many bacterial cells could be further flushed off the towel. The post-wash water was then serially diluted and plated onto mannitol salt plates.

\section{Fingertip Bacterial Transfer}

To simulate personal direct contact with contaminated towels, fingers and palm counts were performed from inoculated towels containing Staphylococcus. As a negative control, uncompromised hands were washed with $95 \%$ ethanol, rinsed with sterile water, air dried, and pressed for three seconds onto a mannitol salt plate. The center template area of the towel was inoculated with the Staphylococcus. The hand was then pressed for three seconds to the center of the inoculated towel template area and pressed for three seconds onto a mannitol salt plate. Fingers and palms were pressed onto two separate mannitol salt plates to ensure potential recovery from all areas of the hand. Hand samples were taken immediately, after one hour, and after 24 and 48 hours post-inoculation. These time frames were chosen because many people take a shower every one or two days. 
Table 1. Staphylococcus aureus replica plate colony forming units (CFUs) recovered from the three types of cotton towels for the three washing conditions. A difference was seen between the bleach and detergent treatments $(p<0.05)$.

Trial 1

Inoculum

$$
2.75 \times 10^{8}
$$

Control

Detergent*

Bleach*

0

Trial 2

Trial 3

Terry

Inner

Outer

31

27

257

44

260

Inner

Outer

182

8

59

0

0

Pima

Inner

Outer

161

273

204

0

0

7

342

8

98

13

11

8

\section{Statistical Analysis}

A three-way parametric analysis of variance (ANOVA) tested the significance of the three washing conditions and three towel types on bacterial retention and viability. A probability level of $p \leq .05$ was used in the analysis. A Tukey's post-hoc test was utilized to determine where the significant differences in washing methods lie. For the fingertip and palm recovery CFUs, a paired student's $t$-test was utilized to see if there were differences.

\section{Results}

\section{Bacterial Recovery}

None of the tubes with no bleach or liquid detergent that were inoculated with $S$. aureus yielded bacterial growth. Similarly, none of the tubes with pre-wash water, none of the tubes with diluted bleach, and none of the tubes with detergent that were inoculated with $S$. aureus yielded bacterial growth. The post-wash water resulted in TFTC for bleach and water samples, but the detergent did grow a few colonies (up to 150) during some of the trials. The post-wash CFUs were added to overall colony counts, but no differences were seen in an ANOVA.

\section{Towels}

Differences were seen between washing methods $(p \leq .05)$, and a Tukey's post-hoc test showed a difference between the bleach and detergent washing conditions. Replica plate colony counts are depicted in Table 1. No bacteria were recovered from towels washed in bleach, and Egyptian cotton seemed to give the highest bacterial recovery counts in water and detergent treatments. We found no statistical differences in colony counts from the towel types ( $p$ $=0.38$ ) or between the inner and outer template areas $(p=0.24)$. Figure 1 shows an upward trend in bacterial recovery from the least to most absorbent towel type, and Figure 2 shows a higher bacterial recovery from the inner template than from the outer template area of each towel type. The number of CFUs recovered from the control and detergent washing conditions were subtracted from the original inoculum concentration and statistical analysis showed there was not a significant difference in retention between the control and detergent. The control still harbors a high number of potentially viable bacterial cells, and we assume the absorbent cotton material, in conjunction with bacterial morphology, helps the bacteria adhere to the material. The detergent appeared to increase the cell recovery by dislodging the bacteria from the towels.

Hands

Hands disinfected with $95 \%$ ethanol revealed no growth on all plates. Direct contact from towels for both the fingertips and palms yielded bacteria too 
numerous to count (TNTC) at immediate examination and at examination one hour postinoculation. Fingertips yielded 75 CFUs at 24 hours, and 38 CFUs at 48 hours. Palms yielded 35 CFUs at 24 hours and 15 CFUs at 48 hours. A paired $t$-test did not show any difference between bacterial viability of the fingertips and palms $(p=0.2)$, but it did show a difference between immediate and one-hour CFUs versus 24 - and 48 -hour recoveries $(p=0.0002)$.

Figure 1. Staphylococcus aureus colony forming units (CFUs) recovered on mannitol salt plates in regard to towel thickness from the three towel types. A $95 \%$ confidence interval is shown.

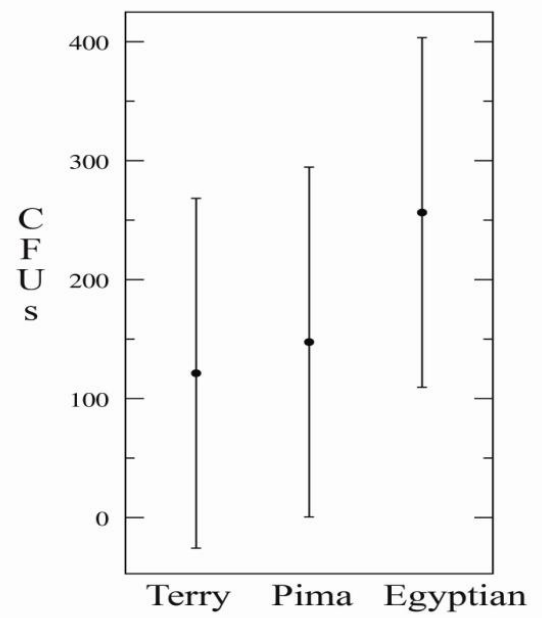

Figure 2. Inner and outer towel template recovery of Staphylococcus aureus (CFUs) from the three towel types. A $95 \%$ confidence interval is shown.

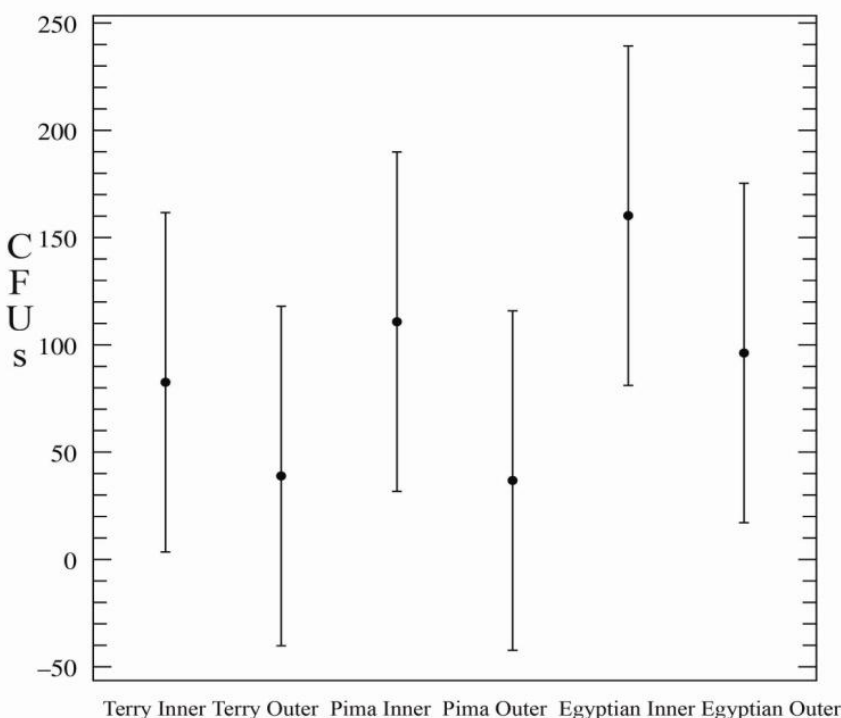

Terry Inner Terry Outer Pima Inner Pima Outer Egyptian Inner Egyptian Outer

\section{Discussion}

Several studies have determined that Staphylococcus could survive for 19 to 21 days on cotton fabrics [6]. Staphylococcus was also reduced when exposed to bleach (69-213 mg/l) [13]. Our replica plate results supported bleach $(p \leq .05)$ as the best washing method for towel disinfection. Although the detergent appeared to allow for greater displacement of bacteria than water alone, bacterial recovery between the two treatments was not statistically significant. Our results also revealed that bacterial displacement over the towel area itself was quite low when subjected to a gentle washing, but we did see higher recovery rates from the inner inoculated template area and lower recovery rates from the outer template areas. Thus, it appears that a gentle wash will not readily displace this bacterium.

Based upon 24- and 48-hour hand contact transfer results, the quantity of potentially viable Staphylococcus left on the towels for 48 hours was the majority of the original inoculum. Although the detergent served as a surfactant to help remove the cells, Staphylococcus was still viable. Our results seem to agree with those of Wilkoff [2] and Neely and Maley [6] in that Staphylococcus can survive for days on cotton fabrics. Our experiment determined that more than $10^{5}$ of viable Staphylococcus can remain on a towel that is washed in either water or detergent, and could feasibly serve as a source of infection both via direct and indirect contact with hands.

Several experimental factors must also be considered. First, inoculum size has been shown to affect quantitative results. Neely and Maley [6] found that an inoculum size between $10^{4}$ and $10^{5}$ exhibited survival on cotton for 16 to 21 days, whereas inoculums of $10^{2}$ survived for 5 to 6 days.

Although Neely and Maley [6] and Gardner et al. [7] found that $3.4 \times 10^{3}$ to $10^{5}$ of bacteria were in wound infections, many people have not had an obvious exposure to Staphylococcus, and yet become nasal carriers and can serve as a disease reservoir. Thus, fewer organisms than $10^{3}$ to $10^{5}$ must be able to colonize people. Although our 24- and 48-hour recovery from directly contacted towels yielded fewer than 100 colonies, these numbers may be high enough to provide colonization in individuals.

Overall, washing towels in bleach was more effective than washing in water or detergent. Thicker, more absorbent towels may harbor more Staphylococcus and for longer times than less absorbent towels, possibly due to the drying time of 
the towel. Staphylococcus was not significantly displaced from the inner inoculated template of the towel during a fifteen-minute gentle wash. Bacterial transfer from towels to hands may occur for at least 48 hours after inoculation of the towel with Staphylococcus. The potential for familial transmission of Staphylococcus aureus via towels is an existing avenue for infection and towel absorbency may play a role in colonization or infection. Thus, using the least absorbent towels and washing them in bleach on a regular basis may help prevent Staphylococcal infections between family members.

\section{Acknowledgments}

We would like to thank William Kirby for laboratory support.

\section{References}

1. Nguyen DM, Mascola L, Bancroft E (2005) Recurring methicillin-resistant Staphylococcus aureus infections in a football team. Emerg Infect Dis 11: 526-532.

2. Wilkoff LJ, Westbrook L, Dixon GJ (1969) Factors affecting the persistence of Staphylococcus aureus on Fabrics. Appl Microbiol 17: 268-274.

3. Grundmann H, Aires-de-Sousa M, Boyce J, Tiemersma E (2006) Emergence and resurgence of methicillin-resistant Staphylococcus aureus as a public health threat. Lancet 368: 874-885.

4. Huijsdens XW, van Santen-Verheuvel MG, Spalburg E, Heck MEOC, Pluister GN, Eijkelkamp BA, de Neeling AJ, Wannet WJB (2006) Multiple cases of familial transmission of community-acquired methicillin-resistant Staphylococcus aureus. J Clin Microbiol 44: 2994-2996.

5. Johansson PJ, Gustafsson EB, Ringberg, H (2007) High prevalence of MRSA in household contacts. Scand J Infect Dis 39: 764-768.

6. Neely AN, Maley MP (2000) Survival of Enterococci and Staphylococci on hospital fabrics and plastic. J Clin Microbiol 38: 724-726.

7. Gardner SE, Frantz RA, Saltzman CL, Dodgson KJ (2004) Staphylococcus aureus is associated with high microbial load in chronic wounds. Wounds 16: 251-257.

8. Leonas KK, Jinkins RS (1997) The relationship of selected fabric characteristics and the barrier effectiveness of surgical gown fabrics. Amer J Infect Control 25: 16-23.

9. Sattar SA, Springthorpe S, Mani S, Gallant M, Nair RC, Scott E, Kain J (2001) Transfer of bacteria from fabrics to hands and other fabrics: development and application of a quantitative methods using Staphylococcus aureus as a model. J Appl Microbiol 90: 962-970.

10. Lankford MG, Collins S, Youngberg L, Rooney DM, Warren JR, Noskin GA (2006) Assessment of materials commonly utilized in health care: implications for bacterial survival and transmission. Amer J Infect Control 34: 258263.

11. Hsieh YL, Merry J (1986) The adherence of Staphylococcus aureus, Staphylococcus epidermidis, and Escherichia coli on cotton, polyester, and their blends. J Appl Bacteriol 60: 535544.
12. Jaska JM, Fredell DL (1980) Impact of detergent systems on bacterial survival on laundered fabrics. Appl Environ Microbiol 39: 743-748.

13. Walter WG, Schillinger JE (1975) Bacterial survival in laundered fabrics. Appl Microbiol 29: 368-373.

Corresponding Author: Anna R. Oller University of Central Missouri, WCM 306, Warrensburg, MO 64093 USA

Phone: (660) 543-4934 Fax:(660) 543-4355

Email: oller@ucmo.edu

Conflict of interest: No conflict of interest is declared. 